\title{
Procesos de razonamiento y comprensión en estudiantes de cuarto grado de educación básica con respecto a la solución de problemas de tipo multiplicativo'
}

\author{
Processes of reasoning and understanding in fourth grade basic \\ education regarding problem solving multiplicative
}

Processos de raciocínio e compreensão em quarto grau do ensino básico sobre problema multiplicativo resolver

Recibido: mayo de 2013

Aceptado: agosto de 2013
John Henry Durango Urrego ${ }^{2}$

Gladys María Rivera González ${ }^{3}$

\section{Resumen}

En los primeros años de la básica, los estudiantes construyen sus propios procesos de razonamiento con respecto a la solución de problemas que involucran las estructuras de tipo multiplicativo, dichos procesos se hacen evidentes a través de explicaciones que ellos construyen. Interpretar estos razonamientos es el propósito central de la investigación, lo cual permite identificar los niveles y dimensiones de comprensión alcanzados por el estudiante a la luz de la referencia teórica de Enseñanza para la Comprensión. El diseño metodológico que orienta la recolección, categorización y análisis de la información es de tipo cualitativo, de igual modo se dan a conocer algunas reflexiones que emergen a partir de la información recolectada.

Palabras claves: Aprendizaje; procesos cognitivos; comprensión; razonamiento; dimensiones; matemáticas escolares; números; operaciones aritméticas; multiplicación; estructura multiplicativa, explicación; otras nociones de Educación Matemática.

\begin{abstract}
In the early years of primary, students build their own reasoning processes with respect to the solution of problems involving multiplicative structures, such processes are evident through explanations they construct. Interpret this reasoning is the central purpose of the research, which identifies the levels and dimensions of understanding achieved by students in the light of the theoretical framework of teaching for understanding. The methodology that guides the collection, categorization and analysis of the information is qualitative, likewise disclosed some thoughts that emerge from the data collected.
\end{abstract}

Keywords: learning, cognition, comprehension, reasoning, dimensions, school mathematics, numbers, arithmetic operations, multiplication,

1 Artículo de Investigación

2 Universidad de Antioquia. Medellin, Colombia. Contacto: jhdurango@ayura.udea.edu.co

3 Universidad de Antioquia. Medellin, Colombia. Contacto: gmriverag@hotmail.com 
multiplicative structure, explanation, other notions of Mathematics Education.

\section{Resumo}

Com base na análise das Diretrizes Curriculares, padrões de concorrência básico e alguns estudos e pesquisas sobre a variação associada ao estudo de trigonometria plana, decidimos aplicar a técnica de análise de conteúdo de alguns livros de décimo grau em relação ao tipo exercícios e "problemas" que são propostos para o estudo das relações trigonométricas, esta análise mostra que geralmente se desenvolve este tema através de expressões algébricas para calcular dados fixos e desconhecidos de um triângulo. Estes resultados mostram a necessidade de desenvolver propostas alternativas que enfatizam as relações exibir relacionamentos "dinâmicas" e funcional entre os ângulos e lados de um triângulo.

Palavras-chave: aprendizagem, cognição, compreensão, raciocínio, as dimensões, a matemática da escola, números, operações aritméticas, a multiplicação, a estrutura multiplicativa, explicação, outras noções de Educação Matemática.

\section{Introducción}

En la formulación y estructuración delos Estándares de competencias y Lineamientos Curriculares del área de Matemática en Colombia, se ha considerado el razonamiento y la comprensión como dos categorías. El primero se ha concebido como un proceso que se encuentra en distintas actividades matemáticas que se dan dentro y fuera del aula (Ministerio de Educación Nacional, 2006, pp. 77-78), mientras que la comprensión se ha abordado como un desempeño flexible que permite al estudiante actuar de manera reflexiva, eficaz, adaptable y generalizable en situaciones reales haciendo uso de conocimientos y experiencias ya vividas (Ministerio de Educación Nacional, 2006, p. 49).

El propósito de este trabajo de investigación es estudiar tanto la evolución de procesos de razonamiento como los niveles y dimensiones de la comprensión en el campo aritmético. La investigación propone la solución de problemas que involucran estructuras de tipo multiplicativo en cuarto grado de Educación Básica Primaria, la pregunta de investigación está orientada a indagar ¿Cómo son los procesos de razonamiento, los niveles y las dimensiones de comprensión de los estudiantes de grado cuarto de Educación Básica Primaria al resolver problemas asociados a las estructuras multiplicativas?

\section{Marco de referencia conceptual}

La presente investigación aborda la categoría del razonamiento desde los planteamientos que establece Balacheff (1987) en donde propone que para lograr la comprensión de los algoritmos y la construcción de las demostraciones es necesario que los maestros descubran la racionalidad que manifiestan los estudiantes acerca del conocimiento que se desea construir; saber cómo funciona y cómo puede evolucionar; basado en estas características racionales, el maestro podrá diseñar actividades que permitan la construcción de nuevos razonamientos, lo que justifica en sí mismo la pertinencia de esta investigación.

Otro elemento que se considera es el rol de lo social asignándole un papel relevante en la construcción del razonamiento pues los estudiantes al compartir con otros sus explicaciones, aclaran y ordenan sus ideas, se convencen unos a otros a través de los argumentos (Balacheff, 1988), de esta manera, asumen cierta responsabilidad frente a los conocimientos que se construyen dentro del aula. 
Teniendo en cuenta que Balacheff plantea tres tipos de procesos de validación, la explicación, la prueba y la demostración y, que los participantes de la investigación son estudiantes que se encuentran en edades entre los nueve y trece años, se dará prioridad a las explicaciones como medio para comunicar las ideas dando cuenta del por qué un argumento es verdadero a través del discurso. La explicación se arraiga en las concepciones del estudiante y en su manera de pensar.

El conocer a profundidad estas explicaciones como exteriorización de sus razonamientos permite establecer dimensiones y niveles de comprensión en los cuales se ubican los mismos, es decir, el razonamiento es un indicador que permite establecer dimensiones y niveles de comprensión, estas dimensiones y niveles se encuentran determinados dentro de la investigación desde la teoría de enseñanza para la comprensión atendiendo a las directrices planteadas por el Ministerio de Educación Nacional a través de los Lineamientos Curriculares (1998) y los Estándares Curriculares (2006), también porque dentro de la teoría se afirma que "la comprensión debe ser construida a partir de la propia experiencia y del trabajo intelectual del estudiante" (Stone, 1999, p. 26) cuando se refiere a la comprensión como una construcción desde el trabajo intelectual se plantea la relación que existe entre razonamiento y comprensión, entendiendo el razonamiento desde Balacheff (1987).

De igual manera, cuando David Perkins analiza las características que hacen parte del desarrollo de la comprensión señala que se debe favorecer el desarrollo de procesos reflexivos como una manera de generar la construcción del conocimiento, proceso que comprende el cómo se formulan las explicaciones y justificaciones alrededor de los conceptos. Las explicaciones y las justificaciones a su vez hacen parte de los procesos de razonamiento y los procesos de validación que plantea Balacheff.

Establecer las dimensiones de comprensión y los niveles en los cuales se encuentran los razonamientos desarrollados por los estudiantes permite diseñar estrategias que conlleven a avanzar en dichos razonamientos y por ende alcanzar un nivel de comprensión cada vez más sofisticado en cada una de las dimensiones.

\section{Metodología}

El proyecto de investigación se ha situado dentro del paradigma cualitativo en consonancia con la pregunta de investigación planteada, las etapas de la investigación y recolección, el análisis e interpretación de la información están basadas en el paradigma cualitativo. Se realiza un estudio descriptivo que permite comprender las particularidades socio-educativas de los estudiantes y la comunidad educativa.

La investigación se realiza con cinco estudiantes matriculados en el grado cuarto, de básica primaria, seleccionados teniendo en cuenta sus habilidades para la solución de problemas de tipo multiplicativo y la realización de las operaciones básicas. Los estudiantes pertenecen a un estrato socio-económico de niveles: uno y dos, el promedio de edad es 10 años. El $60 \%$ son hombre y 40\% son mujeres. No se consideró un mismo número de participantes por sexo. La participación fue voluntaria y no se ofreció ningún tipo de recompensa a los participantes. El tipo de estudio es de caso múltiple (Stake, 1999).

Técnicamente el estudio de caso requiere de la observación participante, de entrevistas abiertas y semi-estructuradas, instrumentos utilizados en el desarrollo de la investigación. El análisis se realiza a través de la triangulación y contrastación de los mismos.

\section{Análisis de los datos}

Los resultados presentados en el presente texto han surgido a partir de la codificación, categorización y tematización de los datos recolectados, de igual forma es necesario aclarar que las respuestas de los estudiantes son consideradas válidas siempre y cuando den cuenta de los procesos de razonamiento que se han llevado a cabo para resolver dicho problema, aun cuando los procedimientos 
que emergen de tales razonamientos sean diferentes a los tradicionalmente esperados, es decir, a través de operaciones directas.

En la dimensión de formas de comunicación se puede observar a través del siguiente ejemplo como los estudiantes en ocasiones también se comunican a través de sus dibujos, estas formas de comunicación le permiten al estudiante crear nuevas relaciones, aclarar sus ideas, organizar la información, como se evidencia en la siguiente actividad.

Entrevistador: ¿Cuántos dulces se necesitan para llenar 7 bolsas que contienen 6 dulces cada una?

Eliza: Toma el lápiz y empieza a dibujar lo siguiente:

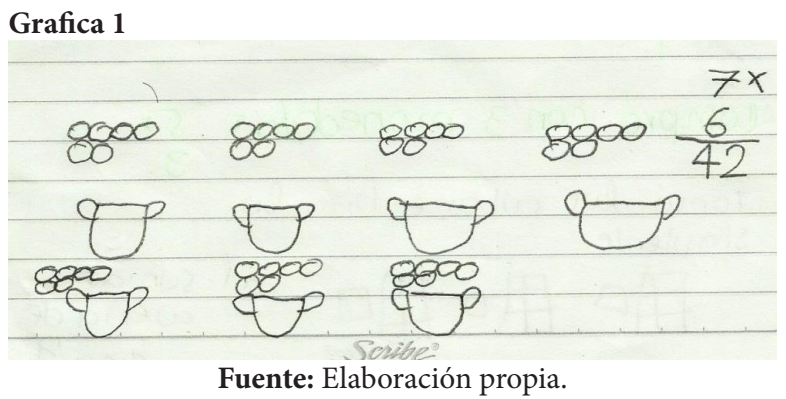

En este sentido Eliza deja notar que en la dimensión de contenido es capaz de moverse de manera flexible en el uso y la aplicación del conocimiento, característica que también se relaciona con la dimensión de praxis, lo anterior refleja la articulación que existe entre las dimensiones de la comprensión.

\section{Conclusiones}

Los estudiantes construyen razonamientos a partir de las explicaciones y argumentaciones que realizan otros compañeros, son activos a la hora de socializar de manera verbal sus razonamientos, dichos razonamientos evolucionan cuando se propician espacios para el debate y la socialización de los procesos que se llevan a cabo en la mente del estudiante.

Generar espacios que les permitan a los estudiantes socializar sus ideas, argumentarlas, realizar conjeturas, crear sus propios procesos algorítmicos y sus propias estrategias de solución desarrolla procesos de razonamiento y al mismo tiempo los niveles y dimensiones de la comprensión.

Finalmente, la presente investigación ha generado nuevas inquietudes que giran alrededor de los procesos de razonamiento de los estudiantes, surgen preguntas como: ¿Cómo inciden las prácticas pedagógicas de los docentes en el desarrollo de los procesos de razonamiento de los estudiantes?

\section{Referencias}

Balacheff, N. (1987). Processus de preuve et situations de validation. Paris: Educational.

Balacheff, N. (1988). Une étude des processus de preuve en mathématique chez des eléves the college. Tesis Doctoral. Grenoble, Grenoble, Francia: Université J Fourier.

Ministerio de Educación Nacional. (1998). Lineamientos Curriculares de Matemáticas. Santefe de Bogotá: Ministerio de Educación Nacional.

Ministerio de Educación Nacional. (2006). Estandares Básicos de Competencias en Matemáticas. En Estándares Basicos de Competencias en Lenguaje, Matemáticas, Ciencias y Ciudadanas (págs. 48-95). Santafe De Bogotá: Ministerio de Educación Nacional.

Perkins, D., \& Blythe, T. (1994). http://www. eduteka.org/AnteTodoComprension.php. Recuperado el 2012, de http://www.eduteka. org/AnteTodoComprension.php : http:// www.eduteka.org/AnteTodoComprension. php

Stake, R. (1999). Investigación con Estudio de Caso. Madrid: Morata.

Stone, M. (1999). La enseñanza para la comprensión. Buenos Aires: Paidos. 